\title{
REGISTRO HISTÓRICO Y EVOLUCIÓN DE LA BARRA ARENOSA DE PUNTARENAS, GOLFO DE NICOYA, COSTA RICA
}

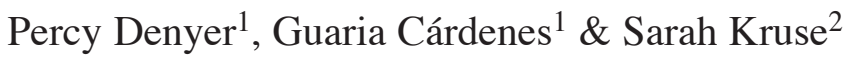 \\ ${ }^{1}$ Escuela Centroamericana de Geología, Universidad de Costa Rica, \\ Apdo. 214-2060 UCR, San José, Costa Rica \\ E-Mail: pdenyer@geologia.ucr.ac.cr,gcardene@geologia.ucr.ac.cr \\ 2 Department of Geology, University of South Florida - Tampa \\ 4202 E. Fowler Ave. SCA 528, Tampa, FL 33620 \\ E-mail: skruse@cas.usf.edu
}

(Recibido 8/8/05; Aceptado 19/10/05)

\begin{abstract}
The Puntarenas sand spit appears in maps from the XVIII century. The $600 \mathrm{~m}$-wide, $7 \mathrm{~km}$-long spit trends E-W with an average elevation of $3 \mathrm{~m}$ above sea level. The spit forms part of the Gulf of Nicoya estuarine system. A geomorphologic analysis of historical maps and aerial photographs reveals systematic growth of the spit over the past 137 years. Puntarenas is part of an estuarine system growing southwards. The sand bar shows lateral growing, basically in La Punta. The spit growth ceased 50 years ago with the onset of coastal engineering. Based on previous photogeological and geophysical studies and new geophysical surveys we identify two paleochannels west of the current Barranca river channel. We infer a NW to SE migration of the Barranca channel, driven by neotectonic activity on the Barranca fault. The lateral growing rate of the Puntarenas spit for the period 1860-1945 (prior to human control) is $14 \mathrm{~m}$ per year; extrapolating this rate back in time yields an origin for the spit approximately 500 years ago.
\end{abstract}

Keywords: Puntarenas, coastal geology, geomorphology, sand bar, historical record.

RESUMEN: La barra arenosa de Puntarenas, conocida históricamente desde el siglo XVIII, tiene dirección E$\mathrm{W}$, una longitud de $7 \mathrm{~km}$, un ancho máximo de 600 y una altura de $3 \mathrm{~m}$; es parte del sistema estuarino del golfo de Nicoya. Se hizo un análisis morfológico de esta barra usando mapas históricos y fotografías aéreas, que revelan un crecimiento sistemático de esta estructura durante 137 años. Se considera que la barra Puntarenas es parte de un sistema estuarino, que muestra un crecimiento hacia el sur, evidenciado por la somerización del canal del estero. La barra arenosa ha presentado un crecimiento lateral principalmente en La Punta, que se ha estabilizado en los últimos 50 años por la actividad antrópica. Con base en estudios fotogeológicos, la geofísica existente y nuevos estudios geofísicos se determinó la existencia de dos paleocanales localizados al oeste del cauce actual del río Barranca, lo que se interpreta como una migración de oeste a este del río, probablemente relacionada con la actividad de la falla Barranca. La formación de la barra arenosa de Puntarenas debe haber estado asociada a la migración del río Barranca y por lo tanto controlada por la tectónica activa en el área. Se calcula que la tasa de crecimiento lateral, durante la formación de esta barra fue de alrededor 14 m/año. Extrapolando esta tasa de progradación se puede inferir que el período de formación de Puntarenas es alrededor de 500 años. Palabras clave: Puntarenas, geología costera, geomorfología, barra arenosa, registro histórico. 


\section{INTRODUCCIÓN}

Puntarenas corresponde con una barra arenosa, desde un punto de vista geológico y, geomorfológicamente se considera una flecha, cuya formación es muy reciente, probablemente durante un período histórico, ubicada en la parte central interna del estuario del golfo de Nicoya (Fig. 1). Es una lengüeta con una dirección prácticamente este-oeste, con su punta al oeste, ligeramente combada al sureste en su extremo occidental. Su longitud es de $10 \mathrm{~km}$ desde la
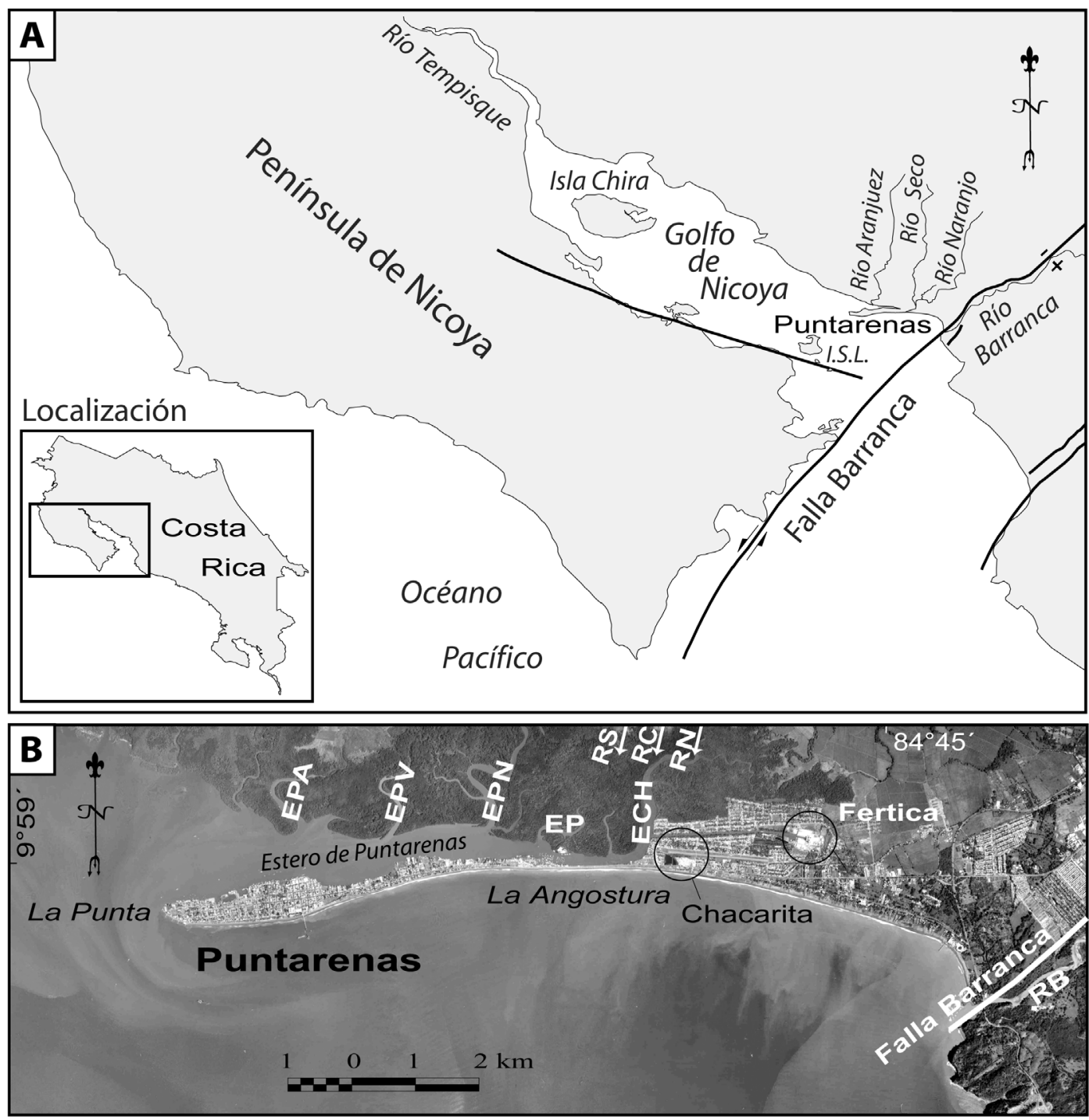

$\downarrow$ Dirección de flujo de río — Falla |l Movimiento horizontal de falla + - Movimiento vertical de falla, + bloque que sube, - bloque que baja

Fig. 1: A) Sistema estuarino del golfo de Nicoya. B) Barra arenosa de Puntarenas (Fotografía aérea del proyecto TERRA, 1997). EPA: Estero Puerto Alto, EPV: Estero Pitahaya Vieja, EPN: Estero Pitahaya Nueva, EP: Estero del Pacífico, ECH: Estero Chacarita, RN: Río Naranjo, RS: Río Seco, RC: Río Ciruelas, RB: Río Barranca, RA: Río Aranjuez, I.S.L.: Isla San Lucas. 
desembocadura del río Naranjo hasta La Punta, su ancho máximo es de $600 \mathrm{~m}$, mientras que la zona más angosta tiene unos $50 \mathrm{~m}$ en La Angostura; su altura máxima es de unos $3 \mathrm{~m}$.

Los sedimentos que la han formado provienen principalmente del río Barranca, cuya desembocadura anteriormente era unos $5,5 \mathrm{~km}$ aguas arriba de la actual, la antigua línea de costa estaba aproximadamente cerca de donde se encuentra en la actualidad los predios de la empresa FERTICA, como lo atestiguan unas 19 paleo-líneas de costa cartografiadas por Madrigal (1971). Esto evidencia una evolución costera en que los ríos Barranca y Naranjo formaron una barra arenosa que inicialmente no estaba unida al continente, que finalmente se unió a tierra firme, hasta construir lo que hoy en día es la ciudad de Puntarenas (Bergoeing, 1983; Madrigal \& Rojas, 1980).

Sus características geológicas implican una gran fragilidad, pues su sustrato arenoso no consolidado es un sistema sedimentológico activo, lo que conlleva la posibilidad de transformaciones rápidas como respuesta a los cambios regionales del sistema estuarino del Golfo de Nicoya. Su constitución hace que esta lengüeta sea susceptible a procesos de licuefacción durante los fenómenos sísmicos que afectan normalmente la región. Además, su condición de zona costera y su poca altura la hace susceptible a la acción de tsunamis (Ortiz et al., 2001). La fragilidad de esta barra arenosa se ha divulgado popularmente en una leyenda que cuenta que el puerto del Callao en Perú sufrió un intenso terremoto ( 28 de Octubre de 1746) que hizo hundirse un gran sector de la ciudad bajo el mar $\mathrm{y}$, se dice que en ese preciso momento apareció Puntarenas; asimismo se dice que Puntarenas desaparecerá y emergerá de nuevo el puerto del Callao (Meléndez, 1977).

El golfo de Nicoya, con una extensión de 80 $\mathrm{km}$ de largo y un ancho entre 15 y $20 \mathrm{~km}$. Es el estuario más importante de la costa pacífica de Costa Rica, se está rellenando con los sedimentos fluviales del río Tempisque y otros ríos, como el Barranca, que desembocan en el golfo. Es un área de hundimiento o estabilidad (Madrigal \& Rojas, 1980), casi todas las geoformas que se observan son de depositación, como lo ratifican los estudios de Marshall \& Anderson (1995) para el extremo suroeste del golfo de Nicoya, donde calculan una subsidencia de $0,5 \mathrm{~m}$ cada mil años. Este golfo, por su forma y su batimetría, puede ser dividido en dos regiones diferentes: la parte interna, rodeado por zonas de manglar, fangosas y con una profundidad menor a los $20 \mathrm{~m}$ y una parte externa, separada por un angosto paso entre la isla San Lucas (Fig. 1) y Puntarenas (Lizano y Vargas, 1993).

Durante la estación seca, las aguas del golfo tienen poca estratificación y una mezcla lenta debido a la poca descarga de agua dulce. Los frentes de salinidad son generados principalmente alrededor de la isla Chira, donde es evidente la influencia de las aguas del río Tempisque. En el resto del golfo la mezcla entre agua salobre y dulce ocurre muy lentamente, dando un comportamiento homogéneo. La época lluviosa se caracteriza por la generación de fuertes gradientes de salinidad tanto en la superficie como a través de la columna de agua, debido a la mayor descarga de los ríos que alcanzan el golfo, por lo que se convierte en un estuario estratificado (Lizano y Vargas, 1993).

Este estudio pretende realizar una revisión histórica de los cambios morfo-sedimentológicos del sistema estuarino de Puntarenas, particularmente de su barra arenosa frontal (Fig.1). Para esto se ha realizado una búsqueda de documentos antiguos y actuales. Se han utilizado tanto mapas como registros escritos y fotografías aéreas, que en total cubren un período de 137 años. Asimismo, se utilizaron métodos geofísicos para comprobar algunas hipótesis.

\section{METODOLOGÍA}

Los mapas históricos resultaron ser de muy variada escala y calidad. Los más antiguos se consultaron en el Archivo Nacional, ahí se fotografiaron y luego se digitalizaron. Para poder hacer las comparaciones se escogieron áreas que estuvieran contenidas en una mayoría de mapas y luego se sobrepusieron digitalmente, con base en puntos de referencia comunes, para poder comparar las morfologías. Un total de 8 mapas históricos contienen detalles de la barra arenosa, 
desde 1860 hasta 1934, posterior a 1945 existen fotografías aéreas en el Instituto Geográfico Nacional, que se consultaron y se digitalizó la línea de costa. La parte del estero y manglares al norte de la barra arenosa no estaba contenida en todos los mapas, así que se hicieron otras figuras comparativas para mostrar la evolución de los manglares con la información disponible. Los datos de corrientes y plumas de sedimentación se obtuvieron a partir de las fotografías aéreas de 1945, 1968 y 1997.

En este estudio se hizo una síntesis de la geofísica hecha anteriormente en la zona (Leandro, 1975 y 1977). Posteriormente realizamos dos campañas geofísicas, en el año 2003, con métodos de resistividad y electromagnetismo. Los perfiles de resistividad fueron realizados con un sistema de 50 electrodos marca Campus, y se interpretaron usando el programa de inversión Res2dinv (Loke and Barker, 1996). El perfil electromagnético se hizo con un equipo EM-34 (Geonics Inc.), con medidas cada $40 \mathrm{~m}$ y una distancia entre bobinas de 10, 20 y $40 \mathrm{~m}$, tanto en modo dipolar horizontal como vertical. La información electromagnética se procesó con el programa EMIX de Interpex Inc., para lo cual se fijaron las profundidades de los contactos en 5 y $20 \mathrm{~m}$ y se hizo una inversión de la resistividad de las capas.

\section{ASPECTOS HISTÓRICOS}

Durante el período de la conquista española, prácticamente no se tienen referencias de la barra de Puntarenas, sin embargo si existen referencias de la zona del estero de Chacarita y el río Naranjo. La hacienda del Palmar, que tenía acceso al estero de Chacarita, en la desembocadura del río Naranjo (Fig. 1), se usó como puerto de los navíos que viajaban a Panamá, desde 1579 hasta la primera mitad del siglo XVIII (González Víquez, 1933), donde también, se construían o reparaban embarcaciones. Las primeras referencias documentales de Puntarenas aparecen en 1765, por parte de Manuel Antonio de Unanué, dueño de la hacienda del Palmar, quien escribió que puede utilizarse como puerto de embarque y desembarque (Meléndez, 1977). Esta primera mención en la documentación de la época parece relacionarse, según Meléndez (1977), con el temporal de octubre de 1962. González Víquez (1933) y posteriormente Meléndez (1977) coinciden en que Puntarenas no existía en la forma como se la conoce hoy, en primer lugar porque no aparece en el levantamiento cartográfico del golfo de Nicoya de Don Gonzalo Fernández de Oviedo y Valdéz, realizado alrededor de 1529 (Meléndez, 1974). Tampoco se habla de Puntarenas ni del estero o, de ningún rasgo geográfico similar en el Derrotero que comunicó el Perú en 1684 para las costas del Pacífico, en el cual sin embargo, si se trata en detalle el golfo de Nicoya. La primera vez que aparece la lengüeta de Puntarenas en un mapa, es en el bosquejo de Don Felipe Molina, que levantó el capitán Belcher, de la marina inglesa, en 1838 (González Víquez, 1933).

El desarrollo antrópico en la barra arenosa de Puntarenas se inicia a partir de 1787 , cuando se iniciaron las actividades portuarias en Puntarenas, pues por ahí se sacaba el tabaco para remitirlo al resto de la región (Meléndez, 1977). En 1814 se habilita como puerto oficial, mediante un decreto. A partir de 1822 empieza a tener importancia gracias al desarrollo de la minería. En 1834 se intentó trasladar el puerto a Caldera, pero finalmente no prosperó esta acción, y poco a poco se convirtió en el puerto más importante y activo del país, hasta llegar a convertirse en la mejor vía para entrar o salir del país en el siglo XIX (Meléndez, 1977). A partir de 1840 reemplaza definitivamente al puerto de Caldera (Oersted, 1940). Alrededor de 1867 el servicio de muellaje era sumamente primitivo, Puntarenas no tenía más que la orilla del estero, los pasajeros tenían que pasar la barra de la punta en botes de remos, en donde solían ocurrir desgracias (Aguilar, 1961). Finalmente, en 1873 se construyó el edificio de la aduana, hoy existente, el palacio municipal y el muelle de hierro. A partir de 1913 se inician labores de dragado en el estero para naves de alto bordo (Aguilar, 1961). Los Baños se construyeron en 1908 por Eduardo Prau y fue un lugar de gran atracción para el turismo (Aguilar, 1961). Esto junto con el advenimiento 
del ferrocarril implicó un notable incremento del turismo josefino, quienes tuvieron en este puerto su lugar de vacación obligatorio, hasta los años 70's del siglo XX, en que se amplía la red vial de Costa Rica y las posibilidades de transporte ampliaron la gama de posibilidades de los costarricenses. A partir de 1981 el puerto oficial del Pacífico pasa a ser el de Caldera, quedando el puerto puntarenense para actividades secundarias y turísticas y por épocas en abandono.

Con respecto a las descripciones geológicas de Puntarenas, A.S. Oersted es el primer naturalista en llegar a tierras costarricenses, entre 1846 y 1848 , y se refiere a Puntarenas como un banco de arena (Oersted, 1940). Attwood (1882) hace un perfil en que se observa la constitución geológica de Puntarenas como arenas finas subyacidas por areniscas calcáreas, probablemente este último se refiere a la Formación Punta Carballo. Además, Attwood (1882) indica que el aporte de sedimentos que forma la barra de Puntarenas proviene del río Barranca.

La primera reconstrucción sobre la evolución morfológica de la barra de Puntarenas fue hecha por González Víquez (1933), en esta comparó los mapas de Kurtze (1860), U.S.S. RANGER (1885) y González (1934) [esta última cita aparece como Salvador González R., 1931 en González Víquez (1933)]. En esta reconstrucción histórica se comprueba el crecimiento de la barra arenosa, textualmente dice: "Y es que Puntarenas se ha ido formando poco a poco con arenas y lodo por los ríos del norte y los que traen los vientos alisios y las corrientes desde la boca del río Barranca." Otros testimonios históricos que indican el crecimiento de La Punta son los de Nicolás Oreamuno, que en 1872 escribió en un documento que aparece en los archivos municipales: “...Como a todos es notorio, porque las cercas al paso que las iba fijando de un manera segura, también tenía que moverlas con frecuencia para ir aprovechando el terreno que el mar iba descubriendo..." (Aguilar, 1961). Lo mismo se puede decir sobre el relleno del canal de La Punta, como se puede leer en González Víquez (1933): "Desde 1914 se hizo por algún tiempo el comercio por Puntarenas, que tenía entonces un buen canal en La Punta, y el estero tenía más profundidad."

\section{GENERALIDADES DEL SISTEMA ESTUARINO DE PUNTARENAS}

A lo largo de todo el contorno del golfo de Nicoya se desarrollan una serie de sistemas estuarinos, cuyos límites varían dependiendo del régimen estacional (Lizano y Vargas, 1993). En su gran mayoría están formados por sedimentos limo-arcillos donde se desarrollan ecosistemas de manglar, en estos las bocas, o zonas de desembocadura de los ríos son relativamente perpendiculares a la línea de costa. Sin embargo, en la parte media del golfo de Nicoya se desarrolla un sistema estuarino único en la zona, el de Puntarenas. Este corresponde con un "Barbuilt Estuary" según la clasificación de Fairbridge (1980). Estos sistemas estuarinos están compuestos por una parte interna con un drenaje tipo reticulado, cuyos materiales son principalmente limo-arcillosos, mientras que en su parte frontal se desarrolla una barra arenosa. La presencia de esta barra arenosa, que en nuestro caso es Puntarenas, permite la protección de la parte trasera (septentrional) del sistema contra el fuerte oleaje y/o las mareas, lo que en el caso particular que se está estudiando ha permitido el desarrollo de un extenso ecosistema de manglar en la parte interior. El ambiente sedimentario de la zona es de tipo mesomareal ( 2 a $4 \mathrm{~m}$ ), lo cual permite el desarrollo tanto del complejo y extenso sistema reticulado como de la barra arenosa.

El sistema estuarino de Puntarenas esta formado por las desembocaduras de los ríos Naranjo, Ciruelas, Seco, y Aranjuez (Fig. 1). Las fracciones granulométricas prevalecientes acarreadas desde las zonas de los esteros Chacarita, Pacífico, Pitahaya Nueva, Pitahaya Vieja, Puerto Alto son limos y arcillas, mientras que las fracciones arenosas son aportadas por la acción de las corrientes marinas.

En relación con su evolución los rasgos generales y macromorfológicos han tendido a permanecer constantes; sin embargo, son de destacar la progradación hacia el sur de los materiales limo arcilloso, cuya consecuencia es la reducción del canal principal de desembocadura en la parte trasera de la barra arenosa (Fig. 2). 

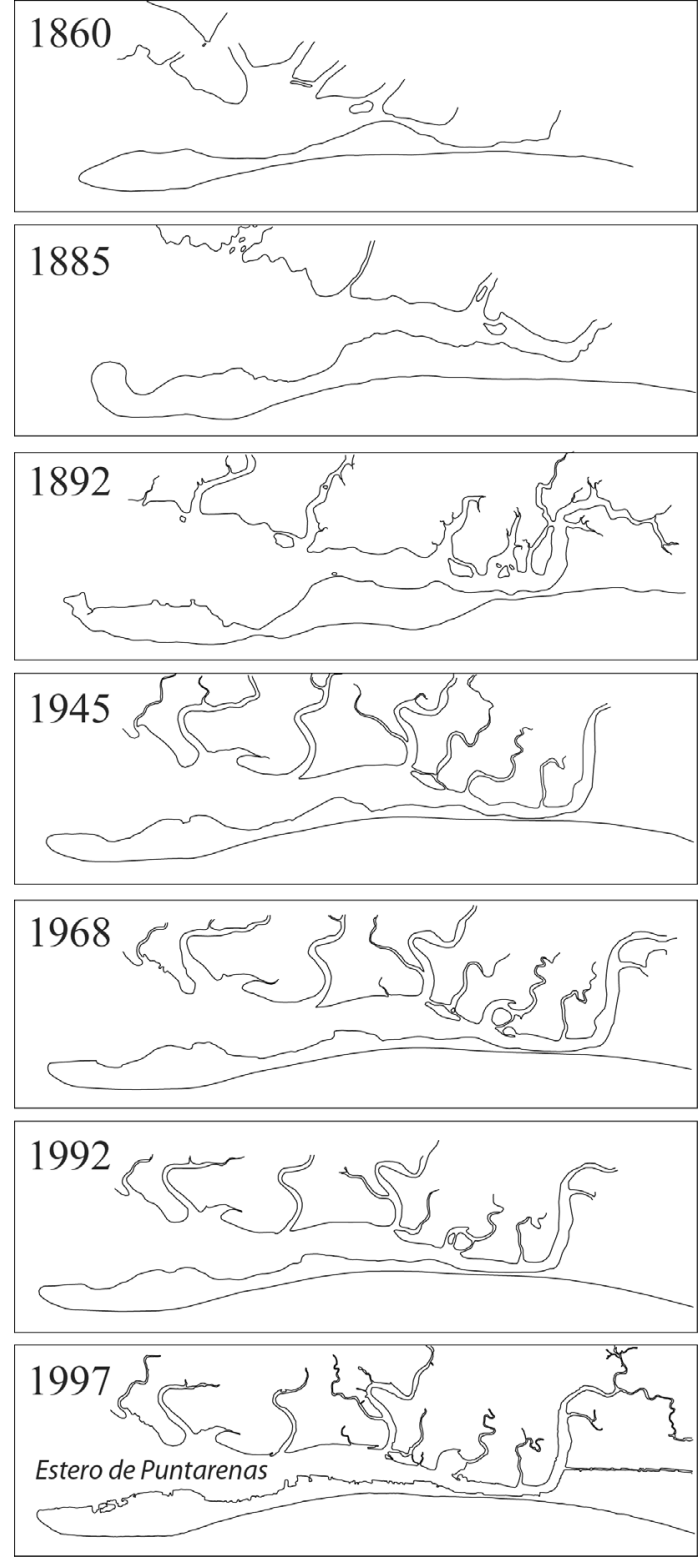

Fig. 2: Reconstrucción morfológica de la barra arenosa de Puntarenas y del sistema estuarino en la parte trasera de la barra. Basado en Kurtze (1860), U.S.S. RANGER (1885), Fradín (1892), IGN (1945, 1968, 1992), TERRA (1997).

\section{EVOLUCIÓN MORFOLÓGICA}

Las barras arenosas generalmente son paralelas a la costa donde se desarrollan, sin embargo, la barra arenosa de Puntarenas tiene una dirección promedio E-W, mientras que la costa aledaña tiene dirección NW-SE. Cabe estacar que esta es la única barra arenosa en Costa Rica con esta característica. Esto se debe a que en algunas ocasiones las barras arenosas no siguen la dirección general de la línea de costa sino que se alinean con respecto a la dirección prevaleciente del oleaje, que en la zona desde Barranca hasta el extremo de la barra de Puntarenas es N-S.

La barra está formada por las fracciones arenosas que son transportadas desde la desembocadura del río Barranca hasta la barra actual. El aporte de sedimentos arenosos por parte de los ríos Naranjo, Ciruelas, Seco, y Aranjuez es despreciable en el proceso de formación de la barra de Puntarenas. La dirección predominante de la corriente litoral en la zona costera desde la desembocadura del río Barranca hasta el extremo más oriental de barra arenosa es E-W, lo cual explica la dirección de esta barra.

En la figura 3 se muestra una reconstrucción morfológica de la barra de Puntarenas desde 1860 hasta 1997, se observa una clara progradación con dirección E-W. En cuanto a su forma general, esta se ha conservado a través de las décadas, se presenta con una parte central formada por dos zonas lobulares en la parte trasera de la barra, una morfología rectilínea en la zona expuesta directamente al oleaje y las mareas, y una zona angosta en el sector este. Las morfologías lobulares de la parte central han tendido, sobre todo desde 1934, a aumentar su tamaño y agudizar su forma. En el sector oeste se han generado los cambios más significativos, con un crecimiento constante y continúo desde 1860 hasta 1948, fecha a partir de la cual la barra baja considerablemente su tasa de progradación hacia el oeste, llegando a ser prácticamente muy baja e incluso nula en los últimos 50 años.

En la figura 4 se muestra un detalle del cambio en el contorno del extremo oeste de la barra. A partir de dicha comparación se puede inferir que desde el inicio del registro en 1860 hasta 1930 la barra tendía a crecer formado un único gancho o brazo hacia el norte. Pero este patrón debió cambiar entre 1930 y 1934, ya que a partir de este momento se erosiona este gancho y más 


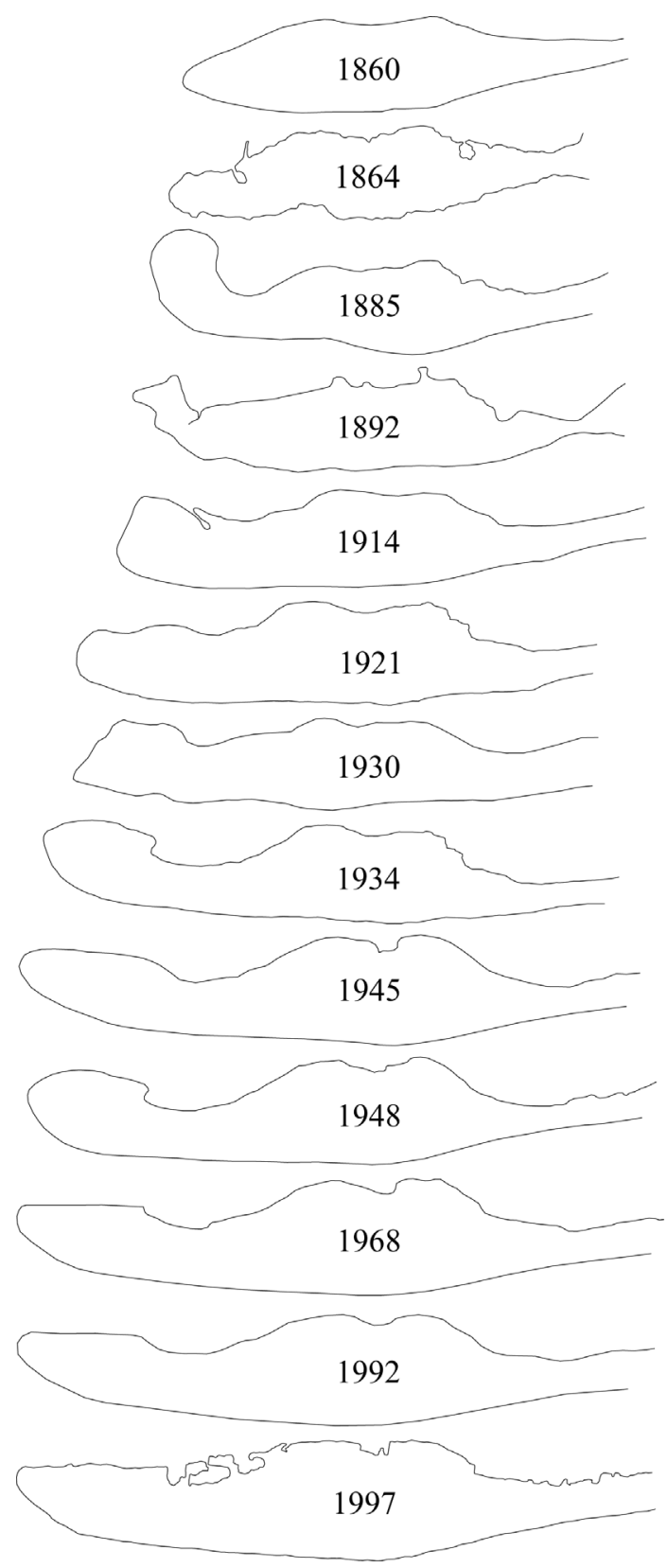

Fig. 3: Reconstrucción morfológica de la barra arenosa de Puntarenas de 1860 a 1997. Basado en: Kurtze (1860), Andrade (1864), U.S.S. RANGER (1885), Fradín (1892), Sprung (1914), Zúñiga (1921), Goedhart \& Brenes (1930), González (1934), IGN (1945, 1948, 1960, 1968, 1992), TERRA (1997).

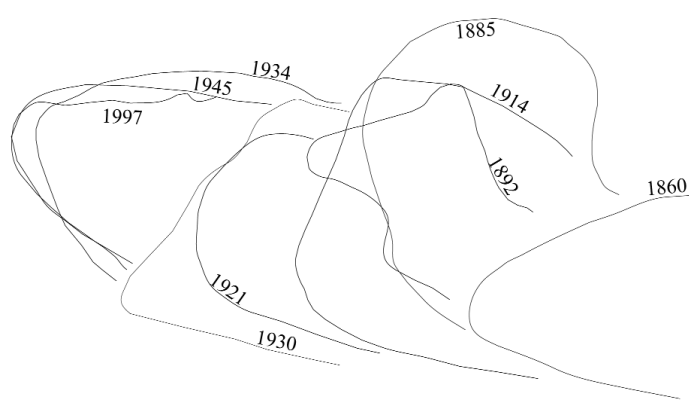

Fig. 4: Detalle de los cambios morfológicos de La Punta. Basado en: Kurtze (1860), U.S.S. RANGER (1885), Fradín (1892), Sprung (1914), Zúñiga (1921), Goedhart \& Brenes (1930), González (1934), IGN (1945, 1992), TERRA (1997).

bien la barra continúa creciendo hacia el oeste, en forma rectilínea. Lo cual probablemente se debió a la actividad antrópica.

Es importante recalcar que cuando las barras arenosas y/o sus alrededores son habitados por seres humanos estos tiende a modificar, a veces de forma drástica, los patrones de erosión/sedimentación, lo que genera fuertes cambios, produciendo lo que se puede llamar una morfología intervenida y, Puntarenas es un caso típico de esta condición. Los dos sitios donde se observa mejor esta intervención son en la zona más angosta conocida como La Angostura y el extremo oeste de la barra, La Punta.

En La Angostura se construyó un muro de rocas que ha impedido la erosión de la parte subaérea. De no haberse construido este muro de rocas es muy probable que la zona subaérea de la barra hubiera desaparecido quedando Puntarenas como una isla, y no como una barra arenosa unida al continente. Como está documentado, desde el año 1827: "El año de 27 pasaron las marejadas a todo lo largo de Puntarenas, se formaron pozos de agua en varias partes, se arruinó la barraca del finado Mollet y otras inmediatas, se abrió un canal, poco más allá de la Chacarita donde pasaba la mar desde media creciente" González Víquez (1933). Esto fue común durante la primera mitad del siglo $\mathrm{XX}$, donde algunos viajeros del tren cuentan que en el paso por La Angostura, el oleaje a veces golpeaba los vagones del ferrocarril. Este es uno de 
esos casos donde el remedio de estabilización costera ha sido eficiente durante un largo periodo de tiempo. Este muro se construyó para contener la erosión y así evitar la destrucción de la línea ferroviaria, así como permitir el libre paso de personas y vehículos, indispensables para el puerto de Puntarenas. La figura 5 presenta un detalle de la zona de La Angostura, desde 1945 hasta 1997. A pesar de lo estrecho de este sector subaéreo de la barra, esta se ha mantenido en conexión subaérea con el continente, durante al menos 250 años.

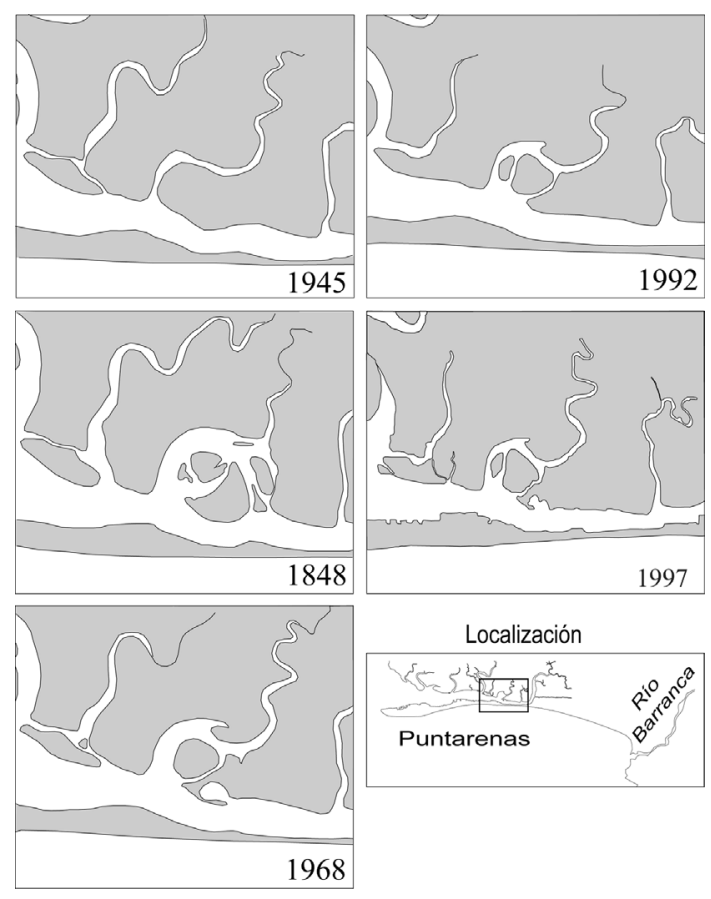

Fig. 5: Cambios morfológicos en la Angostura. Basado en: IGN (1945, 1948, 1968, 1992), TERRA (1997).

En La Punta se pueden documentar mejor las intervenciones humanas en los patrones de erosión y sedimentación. En la sección que da hacia la parte interna del estuario se instaló un atracadero de donde salen barcos pesqueros y de transporte, así como ferries, por lo que se tuvo que adaptar la morfología del fondo por medio de dragados, que se continúan haciendo periódicamente. La morfología de la superficie fue modificada con la construcción de una serie de muros de roca; estos últimos son los responsables de la morfología rectilínea de la zona (ver Figs. 1 y 3). Si no se hubieran realizado estas intervenciones la barra más bien tendría una forma redondeada, o de gancho con dirección hacia tierra, probablemente como la tenía en 1860 (Fig. 3).

Durante la década de 1960 a 1970 se construyeron, en la zona de La Punta, una serie de cinco diques transversales de roca, los cuales se colocaron con el fin de contener los procesos de transporte y sedimentación normales; ya que estos afectaban la forma y la profundidad del atracadero, impidiendo el arribo de los barcos más grandes. En la actualidad dichos diques se encuentran en muy mal estado, quedado solamente la sección de roca que los conecta con la barra. Esto se debe a la falta de un mantenimiento y a las fuertes corrientes que se desarrollan en la zona. A pesar de esto, los materiales que forman los diques permanecen en el fondo, en los alrededores de donde se construyeron los diques originalmente, esto ha sido posible ya que los bloques que formaban los diques eran de tamaños métricos. Esto ha generado una especie de dique subacuático que han permitido la contención de la erosión y el crecimiento del la porción subacuática de la barra.

\section{PATRONES DE SEDIMENTACIÓN}

Se han identificado tres tipos de corrientes que transportan y redistribuyen el material a lo largo de la parte frontal del sistema estuarino de Puntarenas (Fig.6). En primera instancia, las corrientes de marea se encargan de transportar los sedimentos de fracciones finas desde los canales de marea hasta que salen del sistema, hacia la parte externa del golfo de Nicoya. Estas corrientes de transporte tienen una dirección preferencial NNW-SSE, dependiendo de la marea. A pesar de este proceso la sedimentación en la zona frontal del estuario se mantiene en equilibrio dinámico, permitiendo que el efecto neto sea un aumento de los cuerpos sedimentarios intermareales y una 
somerización de los canales. El relleno del canal de La Punta se evidencia en lo expresado en González Víquez (1933): "Desde 1914 se hizo por algún tiempo el comercio por Puntarenas, que tenía entonces un buen canal en la Punta, y el estero tenía más profundidad."

En cuanto al transporte de materiales por las corrientes de oleaje, estas son un factor determinante en la acumulación de fracciones arenosas, las cuales forman la barra de Puntarenas. Las corrientes de oleaje se encargan de traer sedimentos desde la zona marina hasta las cercanías de la barra y depositarlas en la parte frontal de la misma. La dirección preferencial de esta corriente es hacia el NNW.

Por último, las corrientes litorales tienen dos funciones, la primera es tomar los sedimentos acarreados por las corrientes de oleaje y redistribuirlos a lo largo de toda la barra de Puntarenas, mientras que la segunda es acarrear los sedimentos desde la zona de desembocadura del río Barraca hasta la barra. Este último proceso se puede considerar como el principal proveedor de sedimentos de la barra arenosa. Esta corriente tiene una dirección SE-NW en los alrededores de la desembocadura del río Barranca, mientras en la zona de la barra tiene una dirección WSW (Fig. 6).

Las corrientes generadas por el oleaje (Fig. 6) se encargan de transportar las fracciones arenosas desde la parte media del golfo y depositarlas al frente de la barra. Las corrientes litorales permiten la remoción, transporte y resedimentación de las fracciones arenosas al frente de la barra, estas son las principales responsables de la formación y permanencia de la barra de Puntarenas. Por último, las corriente de mareas se encargan de transportar las fracciones más finas (limos y arcillas) que provienen de los esteros Puerto Alto, Pitahaya Vieja, Pitahaya Nueva, Pacífico, y Chacarita hasta la parte media del golfo. Las corrientes de marea son de importancia en el transporte de materiales desde la parte interna del sistema estuarino, sin embargo, estas no son suficientemente efectivas contra el aporte de sedimentos desde el continente, ya que el efecto neto en la parte trasera de la barra es de sedimentación.
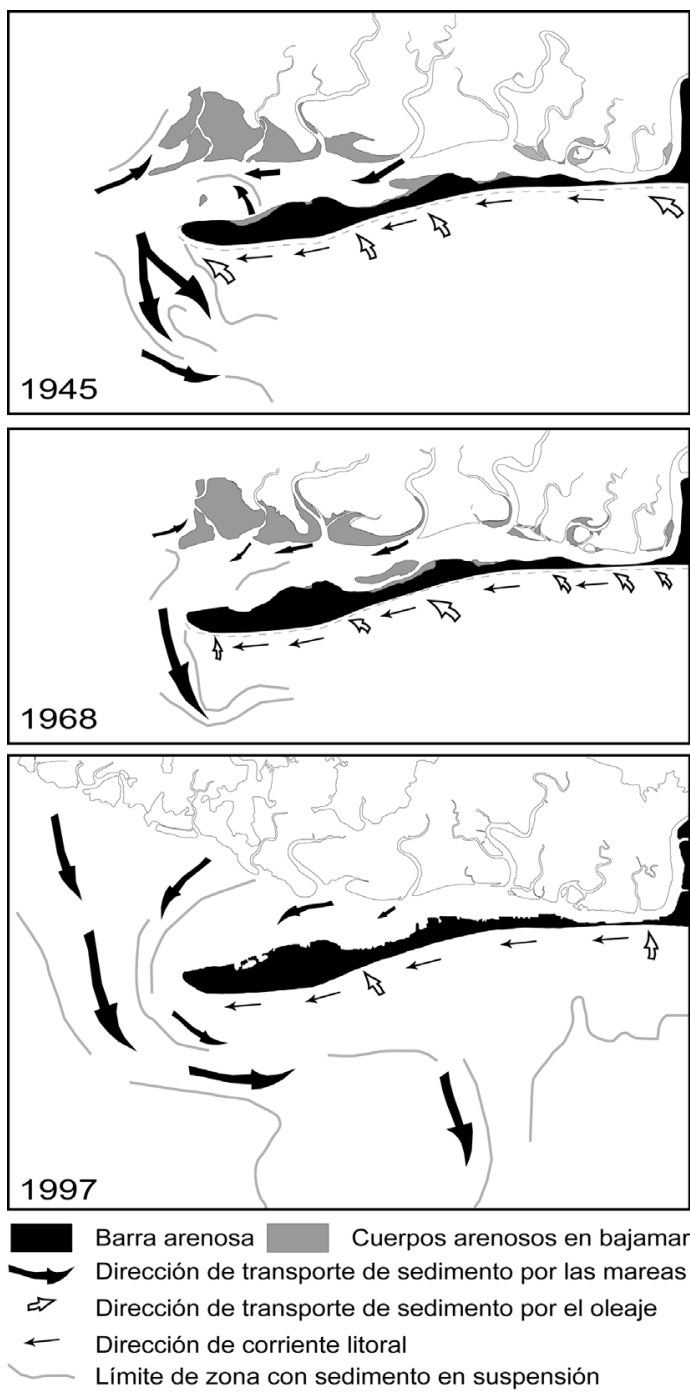

Fig. 6: Distribución de los patrones de transporte de sedimento por el oleaje, las mareas y la corriente litoral. Basado en: IGN (1945, 1968), TERRA (1997).

\section{EVOLUCIÓN MORFOLÓGICA DEL CAUCE DEL RÍO BARRANCA Y LA LÍNEA DE COSTA}

En la zona comprendida entre la desembocadura del río Barranca y la barra arenosa de Puntarenas se han documentado una serie de paleobermas, ya identificadas por Madrigal (1971), las cuales indican que la línea de costa ha 
progradado aproximadamente 2000 metros hacia el sur. Debido a dicha progradación se han generado una serie de depósitos de playa formados por arenas. Las paleo-bermas tienen una dirección WNW, lo cual coincide con la dirección que presentan las facies de playa actuales (Fig.7). En contacto con las facies de playa se presentan las facies de planicie aluvial, y las facies estuarinas.

Madrigal (1971), con base en estudios fotogeológicos indicó la presencia de una serie de paleocanales distribuidos al oeste de la planicie aluvial del río Barranca (Fig. 7). Asimismo, Leandro $(1975,1977)$ usando métodos de gravimetría, resistividad eléctrica y sísmica de refracción, encontró otro paleocanal, también al oeste del río Barranca, a unos $50 \mathrm{~m}$ de profundidad (Fig. 7), en un área en que el basamento está a $100 \mathrm{~m}$.

Anteriormente, se habían reconocido dos zonas de alto contenido de cloruros que penetran en la planicie aluvial, con una dirección noreste (Naciones Unidas, 1975) (Fig. 8). La zona de alto cloruro ubicada más al este se conecta con una zona de alta porosidad, que ha sido interpretada como un paleocanal, con base en los perfiles geofísicos de Leandro (1975, 1977) (Figura 8). Estas zonas de altos valores de cloruros se interpretan como una indentación de la zona de mezcla de agua marina y dulce en capas de alta porosidad, tal y como ocurre con la presencia de sedimentos gruesos tamaño grava. Por lo anterior, la zona occidental (Fig. 8) puede también ser interpretada como un paleocanal. Esta hipótesis es corroborada con las observaciones geomorfológicos hechas por Madrigal (1971), como se aprecia en la figura 8. Para probar esta hipótesis hicimos un perfil geofísico en diciembre del 2003 (Fig. 8), basados en la expectativa de tener resistividades altas (baja conductividad), lo cual correspondería con los parámetros que exhibirían los materiales gruesos de un paleocanal, en donde los materiales gravosos de alta resistividad están en lugar de sedimentos limoarcillosos.

En la figura 9 se muestran los resultados del estudio geofísico. A lo largo del sector sur se hicieron dos perfiles de resistividad con un dispositivo Wenner transversal. En el sector norte se utilizó el método electromagnético para realizar

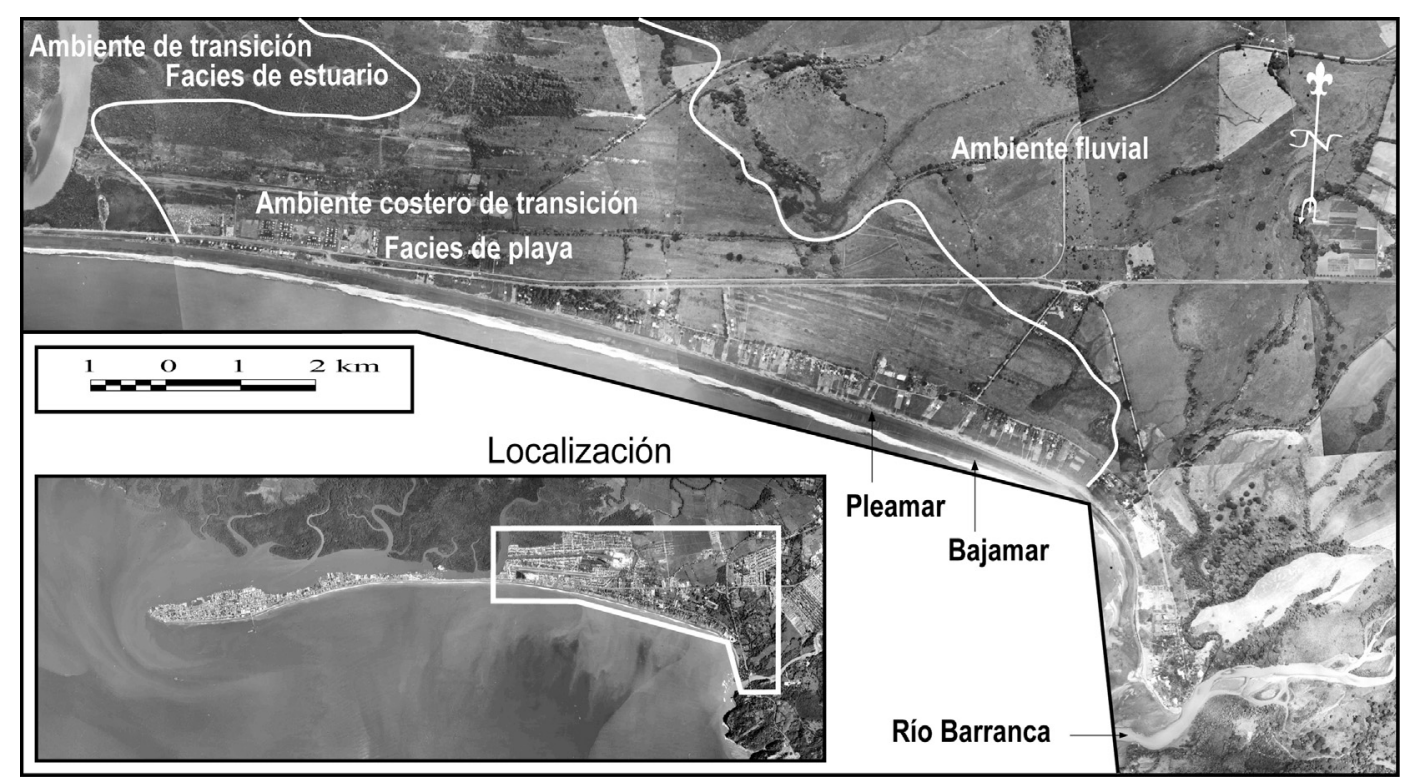

Fig. 7: Distribución de los diferentes ambientes sedimentarios en la zona comprendida entre la desembocadura del río Barranca y el sector oriental de la barra arenosa de Puntarenas. Los lineamientos paralelos a la costa en la zona de facies de playa son paleobermas, que actualmente no es posible observarlas en el terreno ni en las fotos aéreas debido al efecto antrópico. Fotografía aérea (IGN, 1960). 


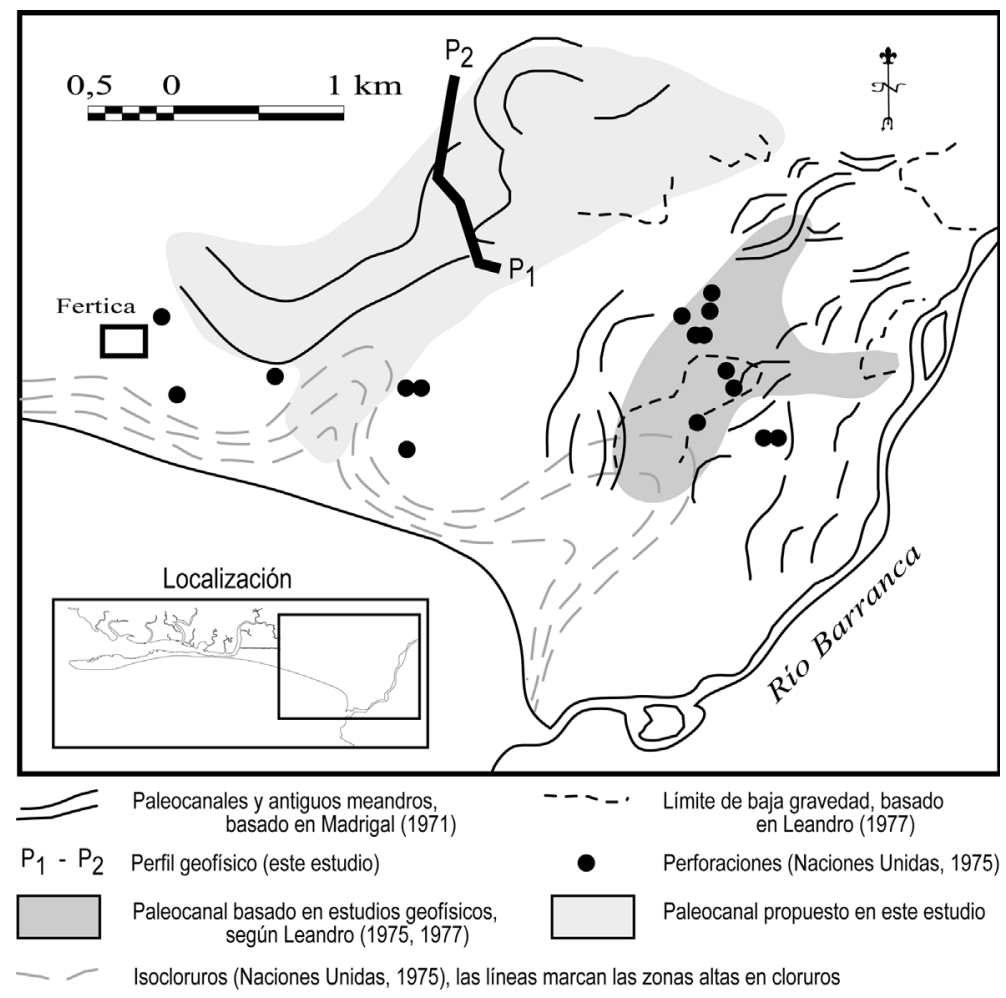

Fig. 8: Paleocanales al oeste del cauce actual del río Barranca.

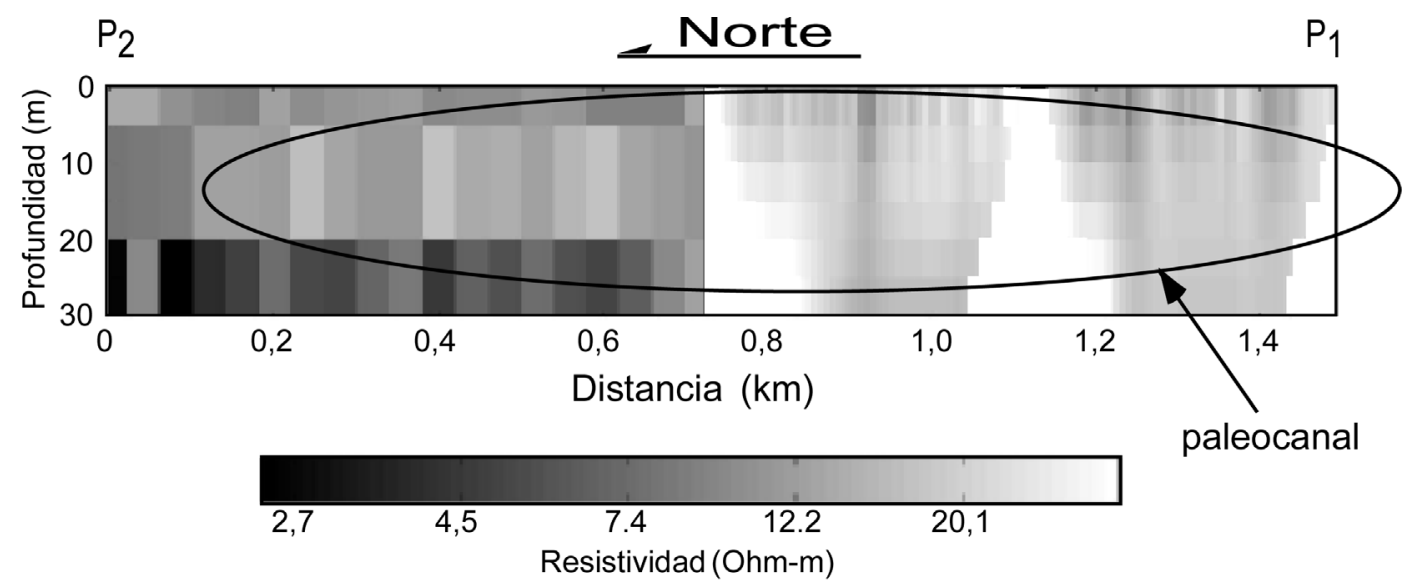

Fig. 9: Perfil de resistividades (Fig. 8). La mitad izquierda (norte) son las medidas electromagnéticas y la mitad derecha (sur) la resistividad (en ambos casos se usó un programa de inversión). La zona sin datos se indica en blanco. La zona de alta resistividad, con base en las observaciones de Leandro $(1975,1977)$, se interpreta como un paleocanal, marcado como un óvalo. Línea de perfil está ubicada en la figura 8. 
un tercer perfil. $\mathrm{Al}$ analizar los datos se determinó una zona de alta resistividad, como se muestra en el óvalo de la figura 9, solo se presenta baja resistividad en los últimos $100 \mathrm{~m}$ del extremo norte del perfil. Con base en estos datos, interpretamos que el paleocanal y la faja de antiguos meandros se extienden unos $2 \mathrm{~km}$ con dirección noreste (Fig. 8), a partir de la indentación de la zona oeste de alto cloruro, terminando en el extremo norte del perfil geofísico.

La existencia de estos paleocauces de gran tamaño al oeste del actual cauce del río Barranca puede interpretarse como una migración de oeste a este del cauce del río Barranca, hasta llegar a alcanzar su posición actual.

El cauce del río Barranca ha migrado de oeste a este, unos $5 \mathrm{~km}$, lo cual no puede ser explicado únicamente por medio de los procesos sedimentológicos en una zona tectónicamente estable, ya que en estos casos la zona de meandros (cauce meándrico) tiende a permanecer en la misma área a través del tiempo, solo se produce abandono y reactivación de canales ya existentes dentro del cauce. Por lo tanto, se considera que esta migración debe estar asociada a fenómenos tectónicos, probablemente en relación con la falla Barranca, que tiene un movimiento siniestral (Denyer et al., 2003), aunque también se ha determinado un movimiento vertical, en que baja el bloque noroeste con respecto al sureste (Fischer et al., 1998), quienes incluso interpretan que este bloque tiene un ligero basculamiento hacia el sureste. Este movimiento diferencial del bloque noroeste de la falla Barranca puede haber causado la migración del cauce y por consecuencia de la desembocadura del río Barranca, hasta la posición en que se encuentra en este momento, prácticamente al pie del bloque de falla.

No se ha datado la actividad de la falla Barranca, ni la edad de los paleocanales que se encuentran al oeste de la planicie aluvial del río Barranca, pero consideramos que puede existir una relación directa entre la formación de la barra arenosa de Puntarenas y la migración de la faja de meandros del río Barranca. Partiendo del supuesto de que las condiciones marinas (oleaje, mareas y corrientes litorales) han permanecido relativamente estables a través del tiempo, se puede suponer que cuando los paleocauces ubicados al occidente estuvieron activos, el flujo de agua aportado por el sistema fluvial debe haber sido lo suficientemente fuerte como para permitir la sedimentación en el frente de la desembocadura; lo que impedía el desarrollo de una barra arenosa en la posición que tiene Puntarenas en la actualidad, y no debe haber sido sino hasta el momento en que el río Barranca se instala en una posición cercana a la actual, que se da la formación de la barra. En estas nuevas condiciones el flujo de agua es menos eficiente transportando y removiendo sedimentos de la antigua desembocadura, debido a que después de la migración solo quedaron pequeños cursos de agua. Estando el río Barranca en la posición actual este aporta el sedimento arenoso suficiente para formar la barra, mientras las corrientes litorales se encargan de depositarlo cerca del estero de Chacarita, formándose así la barra arenosa. Otro factor que puede haber potenciado la formación de la barra arenosa es el aumento en la cantidad de sedimento transportado hacia la costa por el sistema fluvial, como consecuencia de las actividades humanas o por la finalización de la Pequeña Edad del Hielo en el siglo XIX (Lamb, 1995).

\section{DISCUSIÓN Y CONCLUSIONES}

La revisión histórica que se hizo en Archivos Nacionales no ha podido afinar la calidad de la información que ya habían recopilado González Víquez (1933) y Meléndez (1977), definitivamente no hay referencias de la barra arenosa de Puntarenas anteriores al siglo XVIII. Los mapas de Don Gonzalo Fernández de Oviedo, que tanto González Víquez (1933) como Meléndez (1977) ponen como primera evidencia de la no existencia de esta estructura, son esquemas bastante rudimentarios y no dan una perspectiva fehaciente del golfo de Nicoya, por lo que consideramos que perfectamente podrían haber obviado esta delgada lengüeta de arena. Sin embargo, la existencia del puerto y astillero del Palmar, ubicado en el estero de Chacarita implicaba que los navíos tenían que atravesar lo que hoy es parte del 
estero de Puntarenas, lo cual nos hace pensar que anteriormente al siglo XIX Puntarenas pudo haber existido, pero como una barra arenosa separada del continente, o al menos con un canal en La Angostura durante la pleamar.

Un aspecto histórico interesante de recordar, es que a finales del siglo XIX hubo intenciones de modificar el cauce del río Barranca, con el fin de mejorar las condiciones portuarias del estero de Puntarenas, como lo describe el siguiente texto: "Algunos opinan porque se desvíen las aguas del río Barranca, abriéndoles camino para que entren en el Estero y que, aumentando el volumen de las aguas, contribuyan a producir una fuerza suficiente para abrir, limpiar y ahondar el canal que atraviesa la barra de arena" (González Víquez, 1933). Lo cual hubiera podido significar la desaparición de una parte o de la totalidad de la barra arenosa de Puntarenas.

El sistema estuarino de Puntarenas ha tenido un crecimiento hacia el sur durante el período comprendido entre 1860 y 1997. Esto lo sugieren dos hechos, primero el aumento del área ocupada por limos y arcillas de planos intermareales los cuales poco a poco han sido colonizados por vegetación tipo mangle, y segundo la pérdida de profundidad del canal del estero de Puntarenas.

El extremo occidental de la barra arenosa de Puntarenas ha experimentado un crecimiento lateral, al menos desde 1860 (Fig. 3), desde entonces la barra ha aumentado su longitud en $1200 \mathrm{~m}$. Considerando que de 1860 a 1945 los procesos antrópicos no eran tan fuertes como lo fueron después de la segunda mitad del siglo XX, se puede utilizar este periodo de tiempo para calcular la tasa crecimiento lateral en 14 m/año, por lo tanto se necesitarían 411 años para formar los $5,8 \mathrm{~km}$ que tenía la barra en 1860. De ser así, cuando los españoles llegaron a América, apenas comenzaba a formarse la barra de Puntarenas.

La barra arenosa ha mantenido su morfología constante en los últimos 50 años debido a la intervención humana. De hecho, los primeros dragados del estero se iniciaron en $1913 \mathrm{y}$, actualmente se hacen dragados periódicos tanto en la parte trasera de la barra así como en la parte subacuática en el extremo occidental de La Punta, para que pasen embarcaciones de mayor calado, como los ferries, que hacen el servicio de transporte en el golfo de Nicoya.

Los datos geofísicos sugieren la existencia de dos paleocanales al noroeste del actual cauce del río Barranca, uno a 1,5 km (Leandro (1975, 1977) y otro a $3 \mathrm{~km}$ (Madrigal, 1971 y este estudio), lo cual es reforzado por dos indentaciones de cloruros (Naciones Unidas, 1975). Esto se interpreta como una migración de oeste a este del cauce del río Barranca.

La migración del cauce río Barranca de oeste a este, de aproximadamente $3 \mathrm{~km}$ al sureste, puede ser explicada por procesos neotectónicos, donde el bloque noroeste de la falla Barranca ha bajado con respecto al sureste, dejando una ligera inclinación hacia el sureste, que puede haber provocado esta migración del cauce y por consecuencia de la desembocadura del río.

La paleo desembocadura del río Barranca debe haber estado muy cerca de las instalaciones de Fertica. En esta época las aguas del río impedían el desarrollo de una barra arenosa como la actual. Por lo tanto, consideramos que existe una relación directa entre la migración del cauce del río Barranca y la formación de la barra arenosa de Puntarenas y, finalmente ambos fenómenos son una consecuencia de una tectónica activa, que controla los procesos sedimentológicos.

\section{AGRADECIMIENTOS}

Esta publiación es el resultado de los proyectos 113-AS-047 y 113-A6-078 de la Vicerrectoría de Investigación de la Universidad de Costa Rica.

\section{REFERENCIAS}

AGUILAR, H., 1961: Apuntes cronológicos del puerto de Puntarenas. - 143 págs. Inst. Fomento y Asesoría Municipal, San José [Informe interno]

ANDRADE, M., 1864: Plano topográfico de la ciudad de Puntarenas (levantado en pleamar). - Escala original gráfica. San José. [Ubicado en Archivo Nacional de Costa Rica- Serie Mapas y Planos].

ARREDONDO, S., 1995: Evaluación de la vulnerabilidad a la contaminación de los acuíferos costeros Barranca y 
El Roble, Puntarenas, San José. - 76 págs. Univ. de Costa Rica, San José. [Tesis Lic.]

ATTWOOD, G., 1882: Geology of part of Costa Rica. Quarterly J. Geol. Soc. London, 38: 328-340.

BERGOEING, J., 1983: Geomorfología del Pacífico Norte de Costa Rica: explicación del mapa geomorfológico 1: 100 000. - 110 págs. Ed. Univ. de Costa Rica, San José.

DENYER, P., MONTERO, W. \& ALVARADO, G.E, 2003: Atlas tectónico de Costa Rica. - 81 págs. Editorial UCR. San José

FRADÍN, É., 1892: Golfe de Nicoya, estero de Puntarenas. Escala original gráfica. San José.

[Ubicado en Archivo Nacional de Costa Rica - Serie Mapas y Planos].

FAIRBRIDGE, R.W., 1980: The estuary its definition and geodynamic cycle. - En: OLAUSSON, E. \& CATO, I. (ed.): Chemistry and biogeochemistry of estuaries. Ed..Wiley, New York: 1-35.

FISCHER, D.M., GARDNER, T.W., MARSHALL, J.S., SAK, P.B. \& PROTTI, M., 1998: Effect of subducting sea-floor roughness on fore-arc kinematics, Pacific coast, Costa Rica. - Geology, 26(5): 467-470.

GOEDHART, G. \& BRENES F., V.M, 1930: Carta hidrográfica de la entrada al golfo de Nicoya Costa Rica. (Los sondeos son en metros y se refieren al nivel de la marea baja). - Escala original 1:80 000. San José. [Ubicado en Archivo Nacional de Costa Rica- Serie Mapas y Planos].

GONZÁLEZ VÍQUEZ, C, 1933: El puerto de Puntarenas (algo de su historia). - En: Murillo M., M. \& Fenández C., R., 1983: Puntarenas: tres estudios sobre su historia. - Biblioteca Puntarenas No.1, Colegio Universitario de Puntarenas: 40-156.

GONZÁLEZ, D. 1934: Puntarenas y sus barrios. - Escala original 1:4000, San José. (Ingeniero jefe de catastro) y M. Zúñiga A. (Dibujante). [Ubicado en Archivo Nacional de Costa Rica- Serie Mapas y Planos].

IGN, 1945: Fotografías aéreas. Línea CAW 12 (196-198). Escala 1:60 000. Instituto Geográfico Nacional (IGN). San José.

IGN, 1948: Fotografías aéreas. Línea 2-J17 $(17,19,21,23,24,27,29)$. - Escala 1:75 000. Instituto Geográfico Nacional (IGN). San José.

IGN, 1960: Fotografías aéreas [sin numeración visible]. - Escala 1:10 000. Instituto Geográfico Nacional (IGN). San José.
IGN, 1968: Fotografías aéreas. VAP-62 (102-105). - Escala 1:20000. Instituto Geográfico Nacional (IGN). San José.

IGN, 1992: Fotografías aéreas. Rollo 3/ ST-23B (770-772). Escala 1:60 000. Instituto Geográfico Nacional (IGN). San José.

KURTZE, F., 1860: Plano de las operaciones militares ocurridas en Puntarenas por consecuencia de la invasión de Don Juan Rafael Mora de la República de Costa Rica. - Escala 1: 10 000. San José. [Ubicado en Archivo Nacional de Costa Rica- Serie Mapas y Planos].

LAMB, H.H., 1995: Climate, history and the modern world (second edition). - 433 págs. Routledge, London,

LEANDRO, G., 1975: Informe del estudio geofísico con fines hidrogeológicos efectuado en la zona de Barranca - El Roble, mediante los métodos de resistividad eléctrica y refracción sísmica. - 15 págs. Servicio Nacional de Aguas Subterráneas. San José [Informe interno].

LEANDRO, G., 1977: Investigación geofísica del acuífero de Barranca mediante la integración de métodos gravimétricos, sísmicos y de resistividad eléctrica. - 17 págs. Servicio Nacional Nacional de Aguas Subterráneas. San José [Informe interno].

LIZANO, O. \& VARGAS, J.A., 1993: Distribución espaciotemporal de la salinidad y la temperatura en la parte interna del golfo de Nicoya. - Tecnología en Marcha, 12(2): 13-16.

LOKE, M.H. \& BARKER R.D., 1996: Rapid least-squares inversion of apparent resistivity pseudo-sections using quasi-Newton method. - Geoph. Prospecting, 48:181152.

MADRIGAL, R., 1971: Explicación al mapa geológico de un área al nor-oeste del río Barranca. - [número de páginas desconocido, el mapa se consultó en una reproducción que presenta Arredondo (1995), Fig. 3]. Servicio Nacional Nacional de Aguas Subterráneas Informe 58. San José [Informe interno].

MADRIGAL, R. \& ROJAS, E., 1980: Manual descriptivo del mapa geomorfológico de Costa Rica (escala 1:200 000). - 79 págs. Imprenta Nacional-SEPSA, San José.

MARSHALL, J. \& ANDERSON, R.S., 1995: Quaternary uplift and seismic cycle deformation, peninsulas de Niocya, Costa Rica. - Geol. Soc. Amer. Bull. 107(4): 463-473. 
MELÉNDEZ, C., 1977: Costa Rica: tierra de doblamiento en la colonia. - 211 págs. Editorial Costa Rica, San José.

MELÉNDEZ, C., 1974: Gonzalo Fernández de Oviedo. - En: MELÉNDEZ, C.: Viajeros por Guanacaste. Ministerio de Cultura, Juventud y Deportes, Serie Nos Ven no. 4, San José: 23-46.

NACIONES UNIDAS, 1975: Investigaciones de aguas subterráneas en áreas seleccionadas. - 261 págs. Naciones Unidas, San José.

OERSTED, A.S., 1940: La América Central: Investigaciones sobre su flora y su geografía física, resultado de un viaje por los estados de Costa Rica y Nicaragua durante los años 1846-1848. - Rev. Archivo Nacional 4(11-12): 604-623).

ORTÍZ, M., FERNÁNDEZ-ARCE, M. \& ROJAS, W., 2001: Análisis de riesgo de inundación por tsunamis en Puntarenas, Costa Rica. - GEOS. 21(2):108-113.

QUIRÓS V., C., 1983: Aspectos socioeconómicos de la ciudad del Espíritu Santo de Esparza y su Jurisdicción
(1574-1848). - En: Murillo M., M. \& Fenández C., R., 1983: Puntarenas: tres estudios sobre su historia. Biblioteca Puntarenas No.1, Colegio Universitario de Puntarenas: 1-39.

SPRUNG, W., 1914: Sondajes practicados en el puerto de Puntarenas. - Escala original 1:9000. San José.

USS RANGER, 1885: Central America West Coast of Costa Rica Punta Arenas Anchorage (Gulf of Nicoya. - Escala original gráfica, Washington D.C. Hydrographic Office. [Ubicado en Archivo Nacional de Costa Rica- Serie Mapas y Planos].

TERRA, 1997: Fotos aéreas de Costa Rica. - Escala 1: 40 000. Comisión TERRA, San José.

ZÚÑIGA A., M, 1921: Plano de la ciudad de Puntarenas. Costa Rica. - Escala original 1: 2000. San José. [Ubicado en Archivo Nacional de Costa Rica- Serie Mapas y Planos]. 